\title{
Effective Prediction of Thermal Conductivity of Concrete Using Neural Network Method
}

\author{
Jong-Han Lee ${ }^{1)}$, Jong-Jae Lee ${ }^{2), *}$, and Baik-Soon $\mathrm{Cho}^{3)}$
}

(Received June 1, 2012, Revised August 6, 2012, Accepted August 8, 2012, Published online September 20, 2012)

\begin{abstract}
The temperature distributions of concrete structures strongly depend on the value of thermal conductivity of concrete. However, the thermal conductivity of concrete varies according to the composition of the constituents and the temperature and moisture conditions of concrete, which cause difficulty in accurately predicting the thermal conductivity value in concrete. For this reason, in this study, back-propagation neural network models on the basis of experimental values carried out by previous researchers have been utilized to effectively account for the influence of these variables. The neural networks were trained by 124 data sets with eleven parameters: nine concrete composition parameters (the ratio of water-cement, the percentage of fine and coarse aggregate, and the unit weight of water, cement, fine aggregate, coarse aggregate, fly ash and silica fume) and two concrete state parameters (the temperature and water content of concrete). Finally, the trained neural network models were evaluated by applying to other 28 measured values not included in the training of the neural networks. The result indicated that the proposed method using a back-propagation neural algorithm was effective at predicting the thermal conductivity of concrete.
\end{abstract}

Keywords: concrete, temperature distribution, thermal conductivity, prediction, neural network.

\section{Introduction}

Many accidental and environmental factors continue to produce and change heat flow within concrete structures. The magnitude of the temperature variance and resulting thermal behaviors primarily depend on the accuracy of thermal conductivity of concrete (TCC). Since concrete is a composite material composed of water, cement, fine aggregate, coarse aggregate, and other admixtures, the thermal conductivity value changes according to the combination of the concrete compositions including the volume faction and the unit weight of the constituents, and the ratio of water to cement. In addition to the mix proportion and constituents of concrete, many other variables such as quality control, water content, and temperature are directly associated with the value of the TCC. Consequently, the prediction of the TCC is limited and imprecise in the heat transfer and thermal structure analysis of concrete structures.

\footnotetext{
${ }^{1)}$ R\&D Center, POSCO Engineering \& Construction, Incheon 406-732, Korea.

${ }^{2)}$ Department of Civil \& Environmental Engineering, Sejong University, Seoul 143-747, Korea.

*Corresponding Author; E-mail: jongjae@sejong.ac.kr

${ }^{3)}$ Department of Civil Engineering, Inje University, Gimhae 621-749, Korea.
}

Copyright $($ The Author(s) 2012. This article is published with open access at Springerlink.com
The age of concrete hardly affects the change in the TCC except for very early age of about two days due to hydration (Kim et al. 2003). Toyokazu and Yoshiro (1976) also found that the conductivity of concrete slightly increased during the first three days and became almost constant after the age of three days. Thus, this study focused on the value of the TCC in the dry state and accounted for the important variables, such as the mix proportioning, moisture and temperature status, and unit weight of concrete.

The testing methods and procedure for the measurement of the TCC are also complicated and time-consuming. In general, there are three methods of measuring the TCC: the two-linear parallel probe method, the plane heat source method, and the hot guarded plate method (Morabito 1989; Carslaw and Jaeger 1959; Nevile 1995). All the three methods based on similar basic principles require additional efforts to cut the specimen thin and firmly place the thermal probe on/in the specimen, as well as long time control of the dependent and independent variables during the entire testing. Moreover, the conditions of concrete structures exposed to real environmental conditions might differ from those experimentally controlled. Therefore, for the evaluation of temperature distributions and thermal behaviors in the current state of concrete structures, an accurate prediction of the value of the TCC that can account for all these characteristics of the concrete is essential but very complex. For this reason, to investigate the complexity and influences of numerous factors on the TCC, we have employed a backpropagation neural algorithm that emerged as a new alternative to complicated pattern recognition in many fields such as the medical and biochemistry field. 
The neural network, a prediction method for the estimation of the TCC, was constructed and trained using 124 experimental data obtained by previous studies (Kim et al. 2003; Morabito 1989; Harmathy 1983; Yamazaki et al. 1995; Lie and Kodur 1996; Van Geem et al. 1997; Khan et al. 1998; Khan 2002; Kodur and Sultan 2003). Based on their data sets, the developed neural network model was trained with regard to eleven parameters: nine parameters representing the composition of concrete constituents, which were the water-cement ratio, the fine aggregate percentage, the coarse aggregate percentage, the unit water weight, the unit cement weight, the unit fine aggregate weight, the unit coarse aggregate weight, the unit fly ash weight, and the unit silica fume weight, and two parameters representing the state of concrete, which were the temperature of the concrete and the water content in the concrete. Finally, the TCC estimated by the neural network model was compared with 28 randomlyselected measured data not included in the neural network training. As a result, the neural network model, trained by the eleven parameters, accurately estimated the values of the TCC. Therefore, this study demonstrated that the proposed prediction method based on a neural network algorithm could be used as a reliable and effective technique for determining thermal conductivity in the thermal design and analysis of concrete structures.

\section{Construction of Neural Network}

\subsection{Principles of Neural Network}

As problems such as pattern recognition, system identification, and system control became difficult to solve using conventional computing methods, the concept of neural networks was inspired by the biological learning and decision-making process of the human neuron system. In civil engineering, neural networks were well applied to the detection of structural damage (Feng and Bahng 1999), the identification and control of structural systems (Feng and Bahng 1999; Chen et al. 1995), the modeling of material behavior (Adeli and Park 1995), and the proportion of concrete mixtures (Oh et al. 1999). In addition, the compressive strength of concrete (Kim et al. 2004; Kim et al. 2005) was effectively estimated by several researchers, including the authors of this paper, who have applied the neural networks. The main advantage of the neural network approach is easily to perform the predictions, which depend on multiple variables and find difficult to develop an analytical model.

In this paper, we have used feed forward neural networks based on a backward propagation algorithm for the learning of the network. The basic processing elements of the network are artificial neurons and connecting weights, and the complex relationships between input data and corresponding target values are trained to find patterns in data. During the training of the network, the connecting weights are updated in accordance to a particular learning rule until the difference between the predicted values from the feed forward process and the target values meets a tolerance limit. Calculations are conducted from the input of network toward the output data, and errors computed in the output layer are then propagated backward to the input layer. After that, the trained neural network is applied for predicting the outcome of new independent input data.

\subsection{Structure of Neural Network}

To develop the relationships between the TCC and the eleven parameters, this study created a two-layer network with twenty neurons, presented in Fig. 1. Each layer is fully connected to the succeeding layers through the connection weights. The neural network can be expressed as

$$
\text { net }=f\left(\sum_{i} W_{j i} P_{i}+B_{j}\right)
$$

in which $P_{i}$ is the element of input sets, and $W_{j i}$ and $B_{j}$ are the connection weights and biases of the neurons. The input data for the development of the neural network model, which play a key role to reach a satisfactory quality of the neural network approach, were obtained from the literature (Kim et al. 2003; Morabito 1989; Harmathy 1983; Yamazaki et al. 1995; Lie and Kodur 1996; Van Geem et al. 1997; Khan et al. 1998; Khan 2002; Kodur and Sultan 2003).

The sets of input data with initial weights were passed through the network layer, and then the weights and biases were trained using activation functions, which represented a tangent sigmoid function in the first layer,

$$
f^{1}(x)=\frac{\mathrm{e}^{x}-\mathrm{e}^{-x}}{\mathrm{e}^{x}+\mathrm{e}^{-x}}
$$

and a linear transfer function in the second layer,

$$
f^{2}(x)=x
$$

During the training of the network, the weights and biases of the network were iteratively adjusted to minimize the network performance with a back-propagation algorithm. The network performance was based on the mean squared error, MSE, defined as

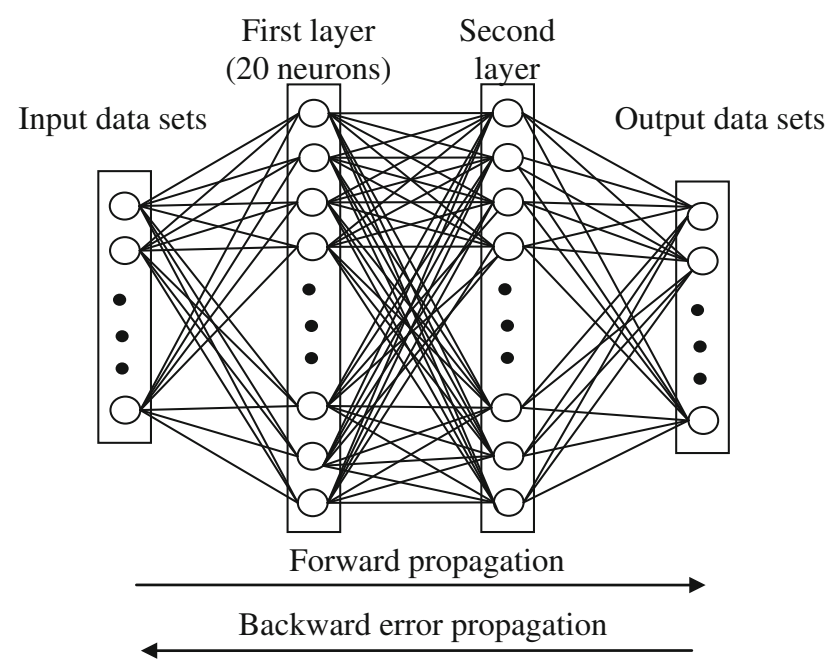

Fig. 1 Structure of the back-propagation neural network. 


$$
\mathrm{MSE}=\frac{1}{N} \sum_{k=1}^{N}\left(T_{k}-a_{k}\right)^{2}
$$

in which $T_{k}$ is the calculated value in the network, $a_{k}$ is the desired thermal conductivity of concrete, and $N$ is the number of the output neurons. The errors associated with desired output data are adjusted in the way that reduces these errors in each neuron from the output to the input layers. The error function was minimized by the Levenberg-Marquardt back-propagation algorithm, which combines the gradient decent and the Gauss-Newton method. When the solution is far from the correct one, the weights are updated in the direction of the negative gradient. When the solution is close to the correct one, the Gauss-Newton method is applied to the training because it is more accurate and faster near an error minimum than the gradient decent method.

The available data sets obtained from the literature were divided into two sets; the training and testing sets. A total of the experimental data sets are 152, of which 124 data (accounting for $80 \%$ of the total data) were randomly selected for the training of the neural network model, and the remaining 28 data (accounting for $20 \%$ ) were utilized for the testing of the network performance. The neural network modeling program was implemented in MATLAB 7.01 software. The number of neurons in the input and the output layers is equal to the number of the input and output data sets. Optimal number of the neurons in the hidden layer was determined to be twenty by training the networks with increases in the number of the neurons.

\subsection{Training of Neural Network}

To train the neural network of the TCC, this research utilized experimental data reported by previous researchers from the 1980's to the 2000's. The training data of the network were composed of 124 sets from Harmathy (1983), Morabito (1989), Yamazaki et al. (1995), Lie and Kodur (1996), Van Geem et al. (1997), Khan et al. (1998), Khan (2002), Kodur and Sultan (2003) and Kim et al. (2003). Table 1 presents samples of the data sets used in the training of the network with eleven parameters: the water-cement ratio, the fine aggregate percentage, the coarse aggregate percentage, the unit water weight, the unit cement weight, the unit fine aggregate weight, the unit coarse aggregate weight, the unit fly ash weight, the unit silica fume unit weight, the temperature of concrete, the water content in concrete.

During the training of the neural network, the weights and biases of the network were updated until its mean squared error was less than a target mean squared error. This study investigated the performance of the neural networks on the basis of four different target errors: 0.10 , $0.05,0.01$, and 0.005 . The prediction performance of the networks was evaluated using the mean squared error (MSE) and the statistical correlation coefficient $(R)$. Figure 2 displays the variations in the mean squared errors and the correlations of the entire training data. The neural network, trained by the 0.10 target error, presents a 0.086 error and a 0.850 correlation between the network outputs and the training sets. As the target errors decreased from 0.10 to 0.005 , the mean squared errors also decreased from 0.086 to 0.003 . In addition to the mean squared errors, the correlations between the network outputs and the training data increased to $0.928,0.983$, and 0.995 in the target errors $0.05,0.01$, and 0.005 , respectively. Furthermore, as represented in Fig. 3, the distributions of the network errors, the difference between the network outputs and the training data sets, showed the decreases as the target errors decrease from 0.10 to 0.005 . The neural networks, trained by the target errors 0.01 and 0.005 , find that most network errors are in the range of -0.1 to $0.1 \mathrm{~W} / \mathrm{m} \mathrm{K}$. Since the difference range is sufficiently accurate in the thermal analysis of concrete structures, this study determines that the two neural networks can be applied as optimum models to the prediction of the TCC.

\section{Comparison of Estimated and Measured Thermal Conductivity of Concrete}

Since the training of the neural networks exhibited similarly good performance in the 0.01 and 0.005 target errors, this study evaluated the applicability of the two neural networks to the estimation of the TCC. To evaluate the trained neural networks, this study randomly selected 28 experimental values not included in the training process of the neural networks from the research results of Harmathy (1983), Yamazaki et al. (1995), Lie and Kodur (1996), Van Geem et al. (1997), Khan et al. (1998), Kodur and Sultan (2003) and Kim et al. (2003). Table 2 shows the details of the 28 experimental values with the eleven parameters: the ratio of water-cement, the percentage of fine and coarse aggregate, the unit weight of water, cement, fine aggregate, coarse aggregate, fly ash, and silica fume, and the temperature and the water content of concrete.

The values of the TCC obtained in the previous experiments were compared with those estimated by the neural networks. Table 3 shows the differences between the measured and estimated values. The results indicate that all of the estimated values based on the eleven parameters show good agreement with the measured ones in both neural networks. In the neural networks with the target error 0.01 , nine estimates matched the measured values, eighteen represented a $0.1 \mathrm{~W} / \mathrm{m} \mathrm{K}$ difference, and the last one showed a $0.2 \mathrm{~W} / \mathrm{m} \mathrm{K}$ difference. The neural network, trained by the target error 0.005 , showed that twelve estimated values were the same as the measured ones and that the remaining values represented the difference of $0.1 \mathrm{~W} / \mathrm{m} \mathrm{K}$. In addition, Fig. 4 presents the mean squared errors of the neural networks and the correlations between estimated and measured thermal conductivity. The errors of the neural networks were found to be quite small: 0.008 in the target error 0.01 and 0.007 in the target error 0.005 . For the correlation values, the two neural networks exhibited extremely high values of 0.985 


\begin{tabular}{|c|c|c|c|c|c|c|c|c|c|c|c|c|c|c|c|c|c|c|c|c|}
\hline 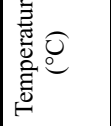 & $m$ & $\nsubseteq$ & $\stackrel{m}{m}$ & $\tilde{\sim}$ & $\stackrel{8}{n}$ & s) & $\stackrel{2}{3}$ & $\stackrel{i}{\infty}$ & i) & i & 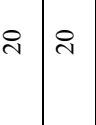 & 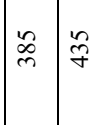 & $\stackrel{\text { ते }}{\circ}$ & 年 & \begin{tabular}{l|l}
$\infty$ & $\infty$ \\
$\infty$ & $m$
\end{tabular} & 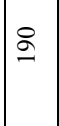 & $\approx$ & $E$ in & $i$ & $\infty$ \\
\hline 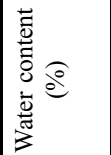 & 0 & 0 & $\begin{array}{lll}0 & 0\end{array}$ & 0 & 0 & $=0$ & 0 & 0 & 0 & 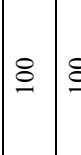 & $\stackrel{8}{8}$ & 0 & 0 & 0 & \begin{tabular}{l|l}
0 & 0
\end{tabular} & 0 & 0 & 0 & o & 0 \\
\hline 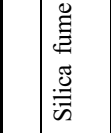 & 0 & 0 & \begin{tabular}{l|l}
0 & 0
\end{tabular} & 0 & 0 & $=0$ & 0 & 0 & 0 & 0 & \begin{tabular}{l|l}
0 & 0
\end{tabular} & \begin{tabular}{l|l}
0 & 0
\end{tabular} & 0 & 0 & \begin{tabular}{l|l}
0 & 0
\end{tabular} & 0 & 0 & 0 & 0 & f \\
\hline 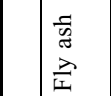 & 0 & 0 & $\begin{array}{lll}0 & 0\end{array}$ & 0 & 0 & 0 & 0 & 0 & 0 & \begin{tabular}{l|l}
0 & 0
\end{tabular} & \begin{tabular}{l|l}
0 & 0
\end{tabular} & \begin{tabular}{l|l}
8 & 8
\end{tabular} & 8 & 8 & \begin{tabular}{l|l}
0 & 0
\end{tabular} & 0 & 0 & 0 & 0 & 0 \\
\hline 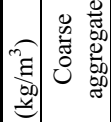 & 0 & 0 & \begin{tabular}{l|l}
0 & 0
\end{tabular} & $\stackrel{\cong}{=}$ & $\cong$ & $\begin{array}{l}0 \\
0 \\
0 \\
0\end{array}$ & đ̊. & ָָ & 0 & 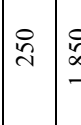 & 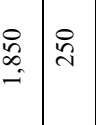 & 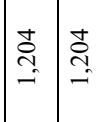 & 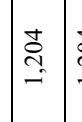 & 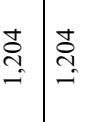 & \begin{tabular}{l|l}
$\stackrel{\infty}{\beth}$ & \multirow{\beth}{\beth}{} \\
$=$ & $=$
\end{tabular} & $\stackrel{\infty}{\beth}$ & 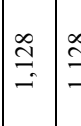 & 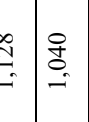 & $\stackrel{9}{+}$ & $\stackrel{\varrho}{=}$ \\
\hline 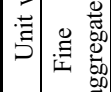 & 0 & o & \begin{tabular}{l|l}
0 & 0
\end{tabular} & $\stackrel{0}{\approx}$ & $\stackrel{\circ}{\approx}$ & $\overline{2} \overline{2}$ & $\bar{\imath}$ & $\overline{2}$ & 3 & $\gtreqless$ & \& & $\hat{i}$ & $\hat{2}$ & $\grave{2}$ & 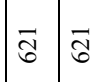 & $\overline{\widehat{\sigma}}$ & $\overrightarrow{\widehat{\sigma}}$ & $\bar{c} \mid \stackrel{2}{2}$ & $\stackrel{2}{2}$ & î: \\
\hline 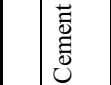 & $\mid$ & בิ & 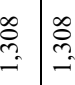 & $\begin{array}{c}\hat{\infty} \\
\infty\end{array}$ & $\stackrel{\infty}{\infty}$ & $\begin{array}{lll}6 & \infty \\
n & \infty \\
n\end{array}$ & ते & ते & 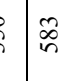 & in & in & \begin{tabular}{l|l}
$\mid I$ & I
\end{tabular} & I & \begin{tabular}{c|c} 
ป & f \\
\end{tabular} & \begin{tabular}{l|l}
$\stackrel{g}{\gamma}$ & $\stackrel{\gamma}{\gamma}$
\end{tabular} & ఫे & के & on & $\ddot{n}$ & d \\
\hline 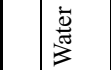 & $\stackrel{\circ}{i n}$ & $\stackrel{\circ}{n}$ & \begin{tabular}{l|l}
$\widetilde{f}$ & $\widetilde{\gamma}$
\end{tabular} & 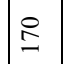 & 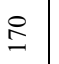 & 2. & $\stackrel{\infty}{\mathscr{\infty}}$ & $\stackrel{\infty}{\infty}$ & 2. & $\cong$ & $\cong$ & 骂 & 骂 & 年| 年 & $\underset{\square}{\square}$ & $\underline{\sigma}$ & $\Xi$ & $\stackrel{\infty}{=}$ & $\stackrel{\infty}{=}$ & $\stackrel{m}{\stackrel{m}{2}}$ \\
\hline 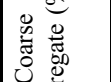 & 0 & 0 & 0 & + & \& & o & $\bar{n}$ & $\bar{n}=$ & 20 & $\approx$ & \begin{tabular}{l|l}
$\&$ & $\infty$ \\
$\&$
\end{tabular} & in $\overline{\text { in }}$ & in & $\vec{n} \bar{n}$ & \begin{tabular}{|l|l}
$\infty$ & $\infty$ \\
$q$
\end{tabular} & $\stackrel{\infty}{\stackrel{\infty}{*}}$ & $\infty$ & 持 & F & F \\
\hline 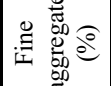 & 。 & 0 & \begin{tabular}{l|l}
0 & 0
\end{tabular} & $\bar{m}$ & $\bar{m}$ & D & ते & ते & $\begin{array}{l}n \\
F\end{array}$ & : & त) & i & i & ¿ & 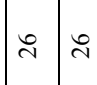 & $\stackrel{\pi}{1}$ & : & $\stackrel{n}{N}$ & $m$ & ते \\
\hline 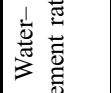 & $\mid$ & $\begin{array}{ll}0 \\
\vdots \\
0\end{array}$ & \begin{tabular}{c|c}
$\tilde{c}$ & $\tilde{c}$ \\
$\hat{o}$
\end{tabular} & $\begin{array}{l}\text { J } \\
0\end{array}$ & $\begin{array}{l}\text { J } \\
0 \\
0\end{array}$ & fif & $\mid \begin{array}{c}0 \\
0 \\
0\end{array}$ & 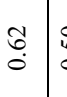 & $\begin{array}{l}n \\
\vdots \\
b\end{array}$ & 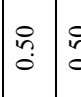 & \begin{tabular}{l|l}
$n$ \\
$\vdots$ \\
0 & 0 \\
0
\end{tabular} & 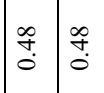 & $\mid$\begin{tabular}{l}
$\infty$ \\
\multirow{0}{0}{} \\
0
\end{tabular} & $\begin{array}{l}\infty \\
0 \\
0\end{array}$ & \begin{tabular}{l|c|c|c|c}
$\hat{o}$ & $\hat{o}$
\end{tabular} & $\hat{m}$ & \begin{tabular}{l|c|c|c|c}
$\hat{o}$ & $\hat{c}$
\end{tabular} & $\frac{n}{b} b_{0}^{n}$ & 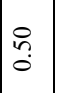 & 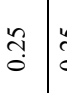 \\
\hline$\underset{b}{0} \frac{\sqrt{a}}{3}$ & : & $\hat{0}$ & $\dot{0}$ & $\exists$ & $\stackrel{m}{=}$ & \begin{tabular}{l|l}
$?$ & \pm \\
- & \pm
\end{tabular} & $\stackrel{0}{-}$ & $\subsetneq$ & 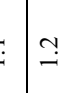 & $\stackrel{7}{\rightarrow}$ & $\Xi \stackrel{\infty}{=}$ & $\exists=$ & $\cong$ & 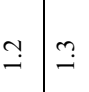 & $\stackrel{\circ}{\circ} \underset{-}{\stackrel{-}{-}}$ & $\stackrel{+}{-}$ & $\because$ & $?=$ & $\stackrel{\sim}{=}$ & 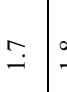 \\
\hline 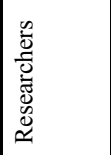 & 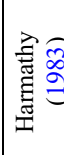 & & & & & & & & & & & 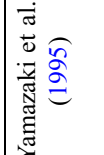 & & & 害言 & & & 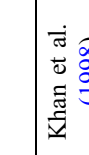 & & \\
\hline
\end{tabular}




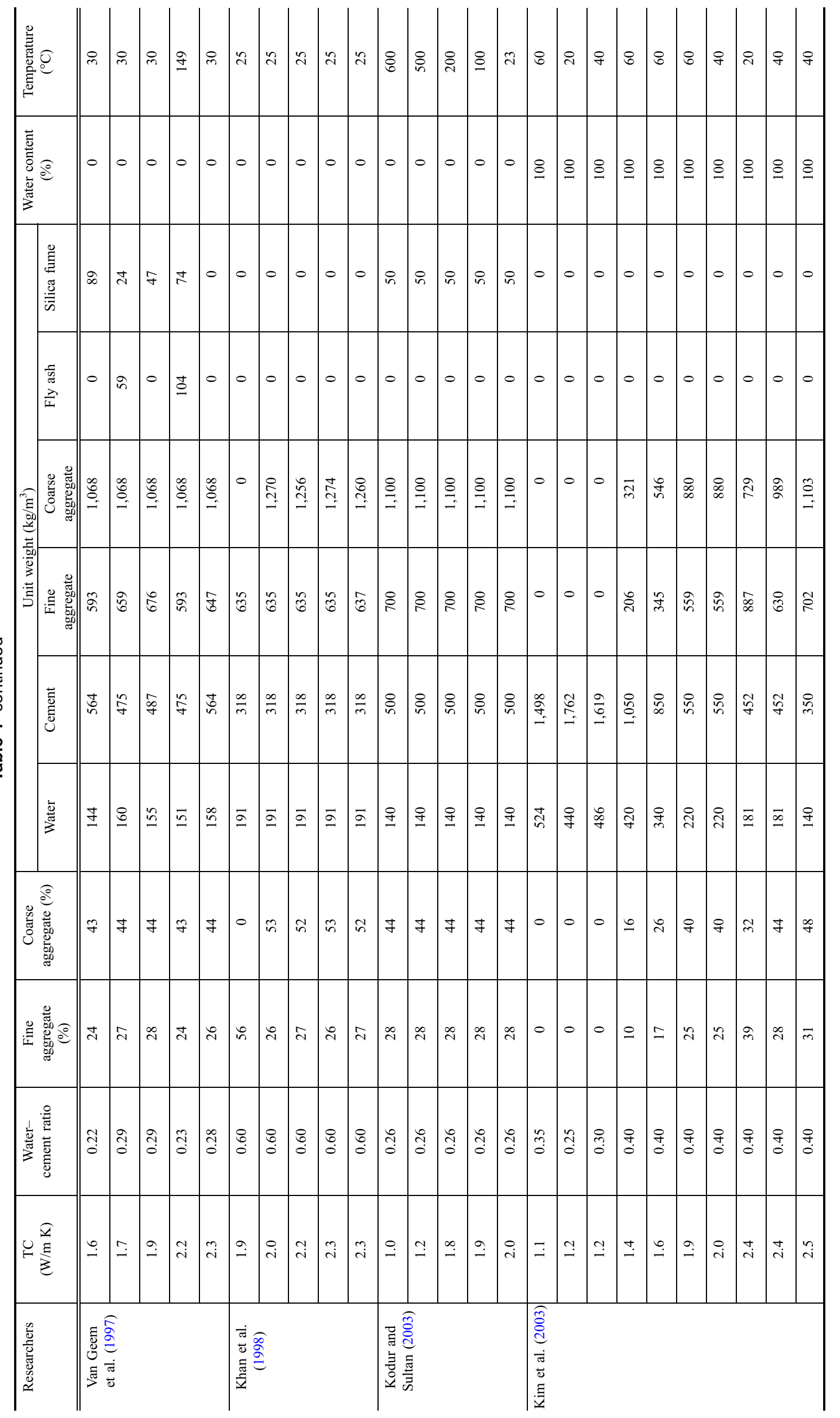



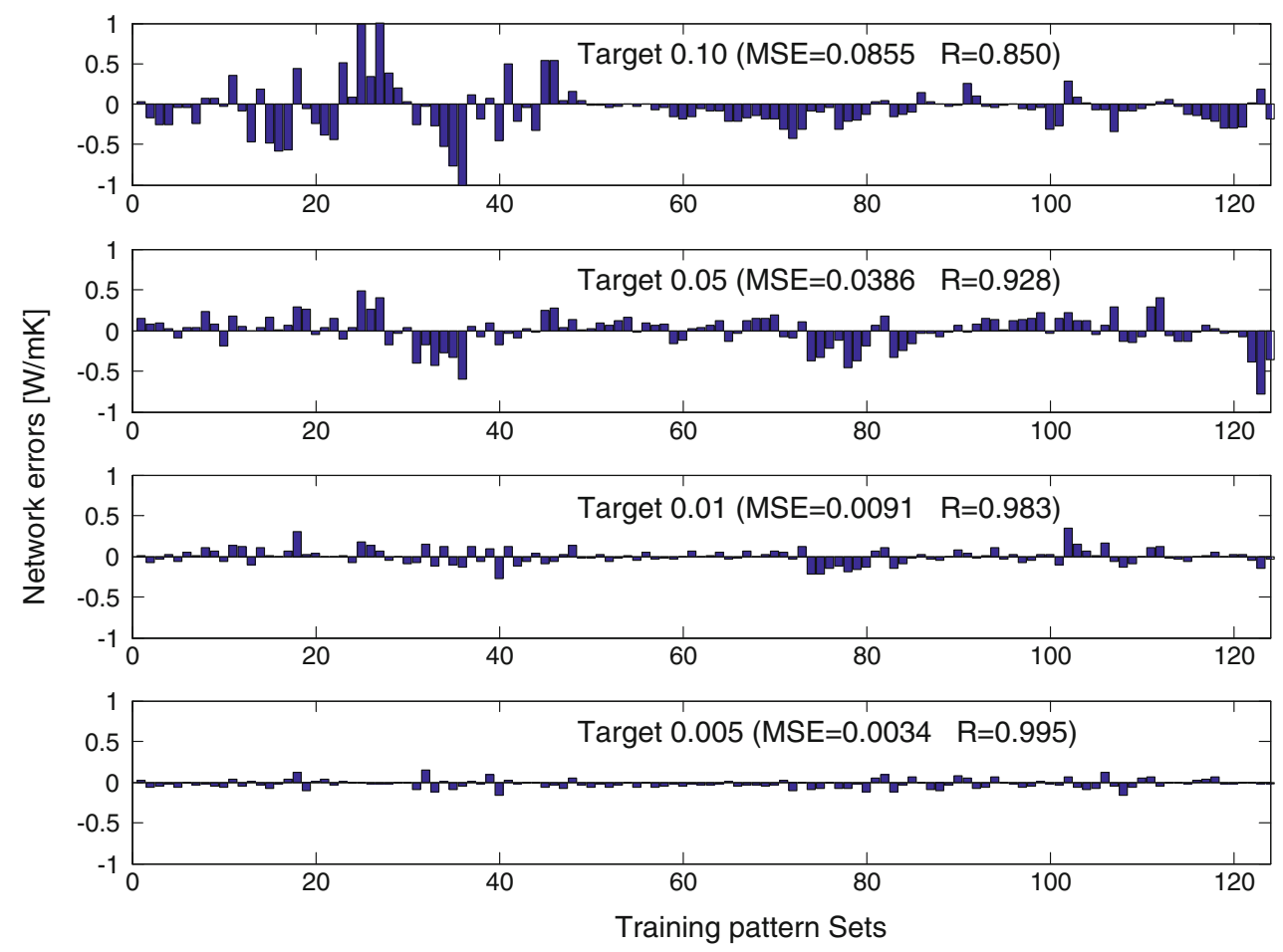

Fig. 2 Variations of mean squared errors and correlations with the training data sets.

(a) Network: Target 0.10

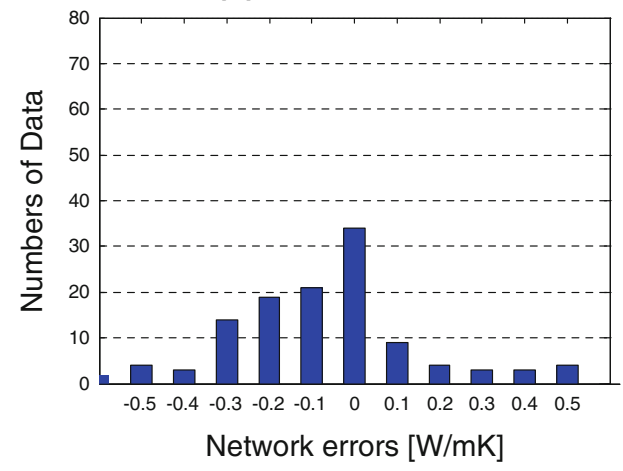

(c) Network: Target 0.01

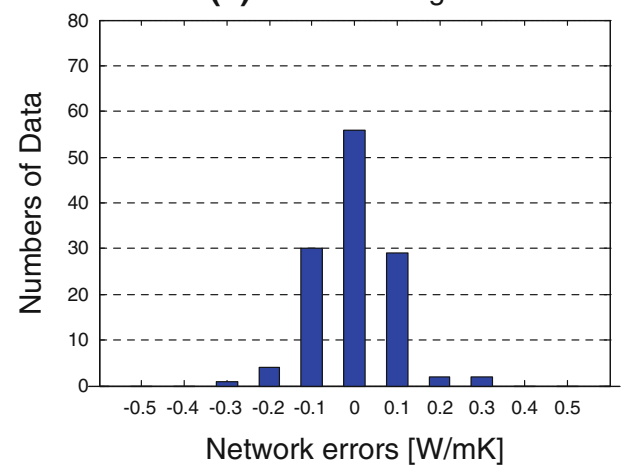

(b) Network: Target 0.05

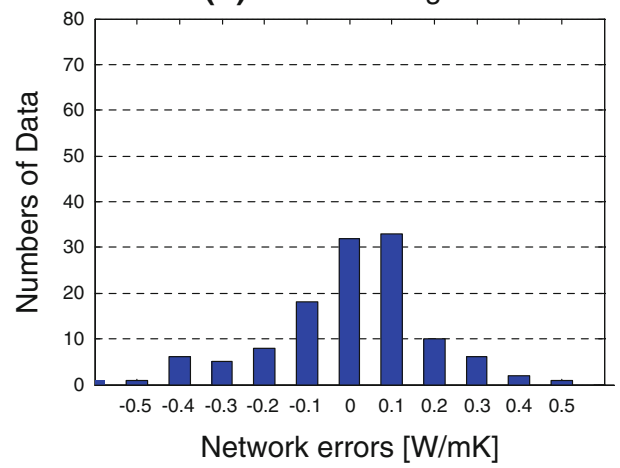

(d) Network: Target 0.005

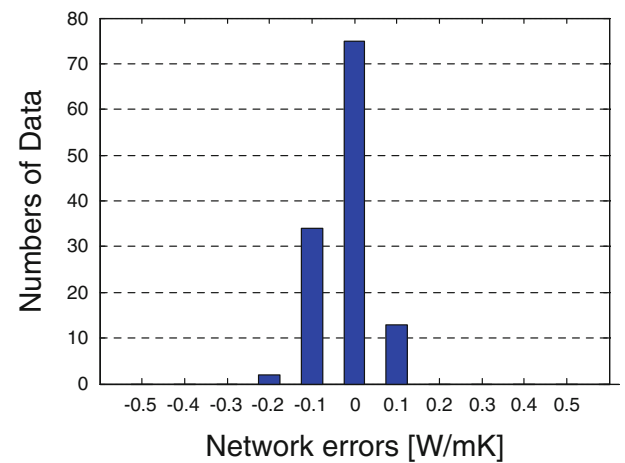

Fig. 3 Distributions of the network errors for the training data sets.

and 0.987 in the target errors 0.05 and 0.005 , respectively. As a result, the proposed neural networks, developed and trained by the eleven parameters, estimated the TCC with high accuracy. Therefore, this estimation method can provide an efficient technique for obtaining the TCC.

\section{Conclusions}

The determination of the TCC is essential to the analysis of its thermal behavior. However, the conductivity value depends on numerous variables, including the composition, 


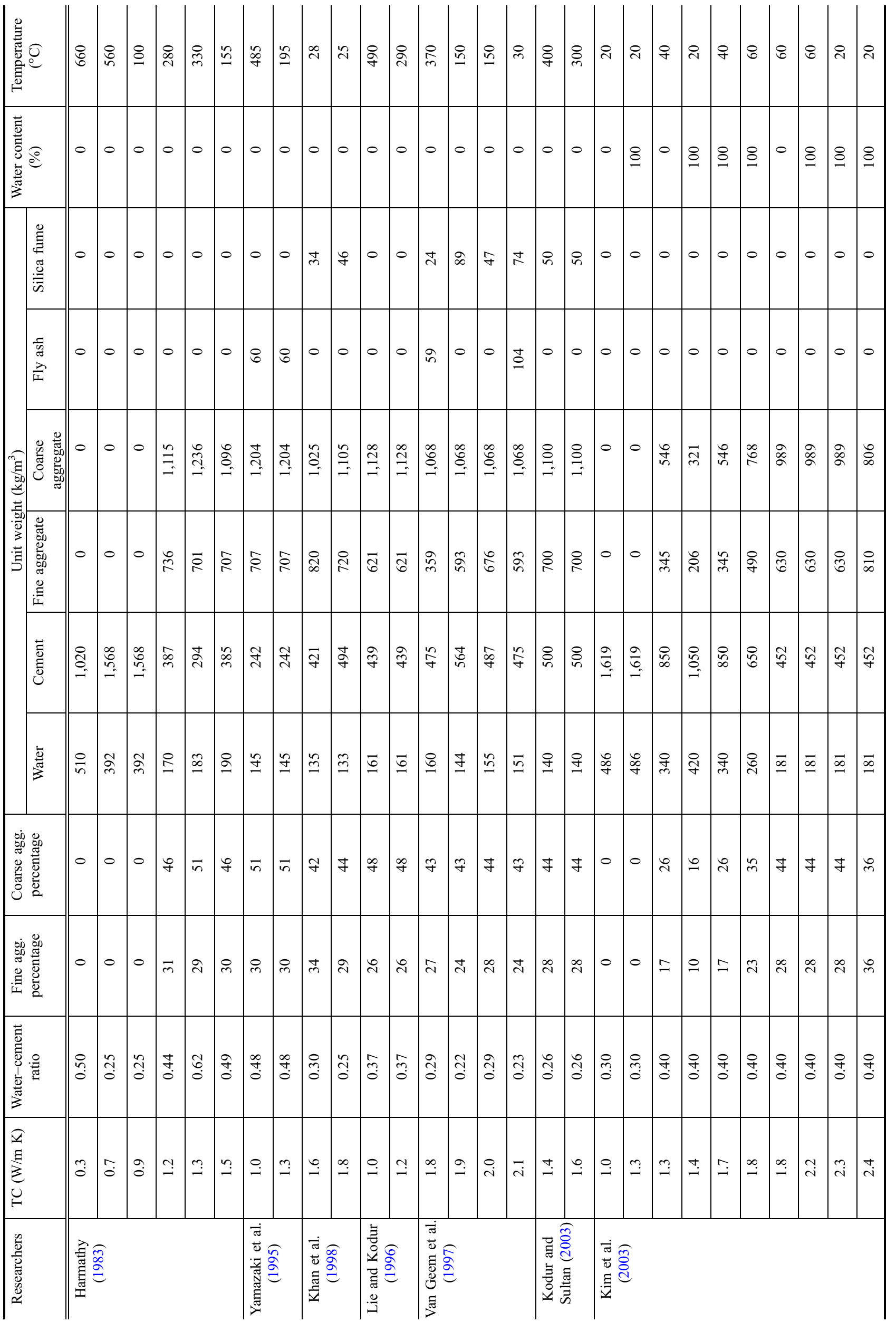


Table 3 Comparisons between the estimated and experimental values.

\begin{tabular}{|c|c|c|c|c|}
\hline \multirow[t]{2}{*}{ Researchers } & \multirow[t]{2}{*}{ Data no. } & \multicolumn{3}{|c|}{ Concrete thermal conductivity $(\mathrm{W} / \mathrm{m} \mathrm{K})$} \\
\hline & & Measured & $\begin{array}{c}\text { Estimated by the target } \\
0.01 \text { network }\end{array}$ & $\begin{array}{c}\text { Estimated by the target } \\
0.005 \text { network }\end{array}$ \\
\hline \multirow[t]{6}{*}{ Harmathy (1983) } & 1 & 0.3 & $0.4(0.1)$ & $0.3(0.0)$ \\
\hline & 2 & 0.7 & $0.8(0.1)$ & $0.8(0.1)$ \\
\hline & 3 & 0.9 & $1.0(0.1)$ & $0.9(0.1)$ \\
\hline & 4 & 1.2 & $1.2(0.0)$ & $1.1(0.0)$ \\
\hline & 5 & 1.3 & $1.5(0.2)$ & $1.4(0.1)$ \\
\hline & 6 & 1.5 & $1.4(0.1)$ & $1.3(0.1)$ \\
\hline \multirow[t]{2}{*}{ Yamazaki et al. (1995) } & 7 & 1.0 & $1.0(0.1)$ & $1.1(0.1)$ \\
\hline & 8 & 1.3 & $1.3(0.0)$ & $1.3(0.0)$ \\
\hline \multirow[t]{2}{*}{ Khan et al. (1998) } & 9 & 1.6 & $1.7(0.1)$ & $1.7(0.1)$ \\
\hline & 10 & 1.8 & $1.8(0.0)$ & $1.7(0.1)$ \\
\hline \multirow[t]{2}{*}{ Lie and Kodur (1996) } & 11 & 1.0 & $1.1(0.1)$ & $1.0(0.0)$ \\
\hline & 12 & 1.2 & $1.3(0.1)$ & $1.2(0.0)$ \\
\hline \multirow[t]{4}{*}{ Van Geem et al. (1997) } & 13 & 1.8 & $1.9(0.1)$ & $1.9(0.1)$ \\
\hline & 14 & 1.9 & $1.8(0.1)$ & $1.9(0.0)$ \\
\hline & 15 & 2.0 & $1.9(0.1)$ & $2.0(0.0)$ \\
\hline & 16 & 2.1 & $2.2(0.1)$ & $2.1(0.0)$ \\
\hline \multirow[t]{2}{*}{ Kodur and Sultan (2003) } & 17 & 1.4 & $1.5(0.1)$ & $1.5(0.1)$ \\
\hline & 18 & 1.6 & $1.7(0.1)$ & $1.7(0.1)$ \\
\hline \multirow[t]{10}{*}{ Kim et al. (2003) } & 19 & 1.0 & $0.9(0.1)$ & $0.9(0.1)$ \\
\hline & 20 & 1.3 & $1.2(0.1)$ & $1.2(0.1)$ \\
\hline & 21 & 1.3 & $1.3(0.0)$ & $1.2(0.1)$ \\
\hline & 22 & 1.4 & $1.4(0.0)$ & $1.5(0.1)$ \\
\hline & 23 & 1.7 & $1.7(0.0)$ & $1.7(0.0)$ \\
\hline & 24 & 1.8 & $1.9(0.1)$ & $1.8(0.0)$ \\
\hline & 25 & 1.8 & $1.7(0.1)$ & $1.9(0.1)$ \\
\hline & 26 & 2.2 & $2.2(0.0)$ & $2.2(0.0)$ \\
\hline & 27 & 2.3 & $2.3(0.0)$ & $2.4(0.1)$ \\
\hline & 28 & 2.4 & $2.4(0.0)$ & $2.4(0.0)$ \\
\hline
\end{tabular}

The values in parenthesis present the differences between the measured and estimated thermal conductivity.

the temperature, and the water content of concrete. Thus, this paper proposed an effective prediction method based on a back-propagation neural algorithm and investigated the influences of all the factors on the TCC. The neural network model was trained using 124 data sets, obtained experimentally in previous research, with regard to eleven parameters: the ratio of water to cement, the percentage of fine and coarse aggregate, the unit content of water, cement, fine aggregate, coarse aggregate, fly ash content, and silica fume, and the temperature and the water content of the concrete.

To determine the optimum neural network model for the estimation of the TCC, this study investigated the variations and distributions of the network errors as the target errors decreased from 0.10 to 0.005 . Since the two neural networks, trained by the 0.01 and 0.005 target errors, showed similarly good performance, the two neural networks were used to estimate the 28 previously measured values not included in the training of the neural networks. The estimated values by the proposed neural networks were very close to the measured values. For the 0.01 neural network, ten estimated values were the same as the measured ones, seventeen differed by $0.1 \mathrm{~W} / \mathrm{m} \mathrm{K}$, and the last one differed by $0.2 \mathrm{~W} / \mathrm{m} \mathrm{K}$. The neural network of the 0.005 target error showed that twelve values were the same as the measured ones and that the 
(a) Network: Target 0.01

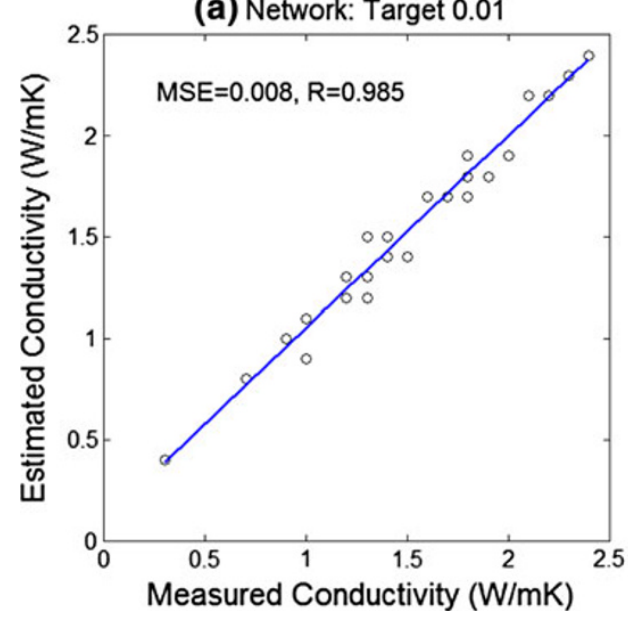

(b) Network: Target 0.005

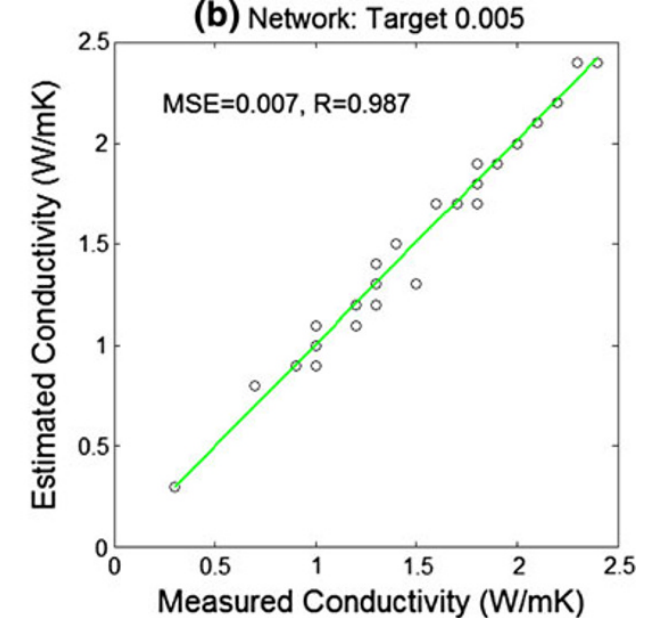

Fig. 4 Correlations of the estimated conductivity with the measured values.

remaining values differed by $0.1 \mathrm{~W} / \mathrm{m} \mathrm{K}$. The differences in the two proposed neural network models are accurate enough to determine the precise temperature distributions of concrete structures. In addition, the correlations between the estimated and measured values were found to be very high: 0.985 and 0.987 in the target error 0.05 and 0.005 , respectively.

In conclusion, the back-propagation neural networks include the influence of multiple factors such as the composition of concrete constituents and the temperature and water content of the concrete in the estimation of the TCC. Based on the estimated results, the proposed network models provide an efficient method for the determination of the TCC. Furthermore, as new data are added to the current training data sets, the performance of the proposed method should dramatically increase.

\section{Acknowledgments}

This work is a part of the research project "Development of an Integrated Design Solution based on Codes and Field Data for the Advancement of the Plant Industry (No. 10040909),” supported by the Korea Government Ministry of Knowledge Economy.

\section{Open Access}

This article is distributed under the terms of the Creative Commons Attribution License which permits any use, distribution, and reproduction in any medium, provided the original author(s) and the source are credited.

\section{References}

Adeli, H., \& Park, H. S. (1995). Neural dynamics model for structural optimization - theory. Computers and Structures, 57(3), 383-390.
Carslaw, H. S., \& Jaeger, J. C. (1959). Conduction of heat in solids (3rd ed.). Oxford: Clarendon Press.

Chen, H. M., Tsai, K. H., Qi, G. Z., Yang, C. S., \& Amini, F. (1995). Neural network for structure control. Journal of Computing in Civil Engineering, 9(2), 168-176.

Feng, M. Q., \& Bahng, E. Y. (1999). Damage assessment of jacketed RC columns using vibration tests. Journal of Structural Engineering, 125(3), 265-271.

Harmathy, T. Z. (1983). Properties of building materials at elevated temperatures, DBR Paper No. 1080. National Research Council of Canada Division of Building Research.

Khan, M. I. (2002). Factors affecting the thermal properties of concrete and applicability of its prediction models. Building and Environment, 37(6), 607-614.

Khan, A. A., Cook, W. D., \& Mitchell, D. (1998). Thermal properties of transient thermal analysis of structural members during hydration. ACI Materials Journal, 95(3), 293-303.

Kim, K. H., Jeon, S. E., Kim, J. K., \& Yang, S. C. (2003). An experimental study on thermal conductivity of concrete. Cement and Concrete Research, 33, 363-371.

Kim, J. I., Kim, D. K., Feng Maria, Q., \& Yazdani, F. (2004). Application of neural networks for estimation of concrete strength. Journal of Materials in Civil Engineering, 16(3), 257-264.

Kim, D. K., Lee, J. J., Lee, J. H., \& Chang, S. K. (2005). Application of probabilistic neural networks for prediction of concrete strength. Journal of Materials in Civil Engineering, 17(3), 353-362.

Kodur, V. K. R., \& Sultan, M. A. (2003). Effect of temperature on thermal properties of high-strength concrete. Journal of Materials in Civil Engineering, 15(2), 101-107.

Lie, T. T., \& Kodur, V. K. R. (1996). Thermal and mechanical properties of steel-fibre-reinforced concrete at elevated temperatures. Canadian Journal of Civil Engineering, 23(2), 511-517.

Morabito, P. (1989). Measurements of the thermal properties of different concretes. High Temperatures-High Pressures, 21(1), 51-59. 
Nevile, A. M. (1995). Properties of concrete (4th ed.). UK: Longman.

Oh, J. W., Lee, I. W., Kim, J. T., \& Lee, G. W. (1999). Application of neural networks for proportioning of concrete mixes. ACI Materials Journal, 96(1), 61-67.

Toyokazu, S., \& Yoshiro, M. (1976). Thermal properties of mortar and concrete in hardening process. Transactions of the Architectural Institute of Japan, 241, 1-8 (in Japanese).
Van Geem, M. G., Gajda, J., \& Dombrowski, K. (1997). Thermal properties of commercially available high-strength concretes. Journal of Cement, Concrete, and Aggregates, 19(1), 38-54. Yamazaki, N., Yamazaki, M., Mochida, T., Mutoh, A., Miyashita, T., Ueda, M., Hasegawa, T., Sugiyama, K., Hirakawa, K., Kikuchi, R., Hiramoto, M., \& Saito, K. (1995). Structural behavior of reinforced concrete structures at high temperatures. Nuclear Engineering and Design, 156(1-2), 121-138. 